\title{
Understanding viral host interactions that modulate nuclear transport and innate immunity
}

\section{S. Tsimbalyuk' ${ }^{1}$, K.M. Smith ${ }^{1}$, M.R. Edwards² ${ }^{2}$ E.M. Cross ${ }^{1}$, J. Batra ${ }^{2}$, T.P.Soares da Costa ${ }^{3}$ D. Aragão ${ }^{4}$, C.F Basler ${ }^{2}$, J.K. Forwood ${ }^{1}$}

${ }^{I}$ School of Biomedical Sciences, Charles Sturt University, Wagga Wagga, NSW, 2678, Australia.

${ }^{2}$ Center for Microbial Pathogenesis, Institute for Biomedical Sciences, Georgia State University, Atlanta, GA, 30303, USA.

${ }^{3}$ Department of Biochemistry and Genetics, La Trobe Institute for Molecular Science, La Trobe University, Melbourne, VIC, 3086, Australia.

${ }^{4}$ Australian Synchrotron, Australian Nuclear Science and Technology Organisation, 800 Blackburn Road, Clayton, VIC, 3168, Australia.

\section{jforwood@csu.edu.au}

RNA viruses such as coronaviruses, flaviviruses, and henipaviruses represent major international health threats. Whilst these viruses replicate in the cytoplasm, they encode accessory proteins that target the host nuclear transport machinery to suppress innate immune pathways. Specifically, these virus proteins target the nuclear import receptor importin-a (IMPa) and inhibit host immune responses from entering the nucleus and triggering interferon (IFN) release. This immune evasion strategy is a critical component of virus pathogenicity, yet details of these interactions (including mechanism(s) of binding specificity with IMPa isoforms) remain unresolved. Here we describe the interfaces between these viral immune regulatory proteins and specific IMPA host receptors as targets for development of novel antivirals.

Keywords: Nuclear transport; Henipavirus; Hendra; Nipah; Importin 